\title{
Study of Recent Development in Medical Image Processing Using Computer Visualization Based Method
}

\author{
P.Alamelu ${ }^{1}$, P.Pragadeshkumar ${ }^{2}$, V.Annapoorani ${ }^{3}$ \\ ${ }^{1}$ AVS Engineering College, Salem, Tamil Nadu, India \\ ${ }^{2 \& 3}$ Mahendra Institute of Technology, Namakkal, Tamil Nadu, India
}

\begin{abstract}
The information extraction development of medical image, for instance Liver picture from exact camera, is complete of complexities and noises. As a result, cost used up on such processing like time and resources is high, particularly for big and complex quantity of information. This manuscript uses computer visualization-based methods to address the challenges. This method mainly includes four stages with unlike algorithms to deal with the medical liver images. Initially, smoothing algorithm is used to reduce the noise. Secondly, filtering algorithm is used for image study so as to recognize the target region. Thirdly, further enhancement algorithm is used to figure out the image features within the target area, finding out the basic outline of the image. Eventually, reduction algorithm is utilized to convert the original images into more smooth and precise pictures. Tentative results demonstrate that computer visualization-based medical image processing algorithm can accurately extract the relevant data and achieve better results, comparing with the traditional image processing method.
\end{abstract}

Keywords: Medical Image Processing; computer Visualization; Algorithm; Liver Image; Digital Image

\section{INTRODUCTION}

Computer visualization is a product of a high degree of integration of science and technology. As a regulation, computer visualization is concerned after artificial systems theory science. It is extracted from the picture representation.

The picture data may be obtained by using a variety of ways, such as a video sequence, from the plurality of cameras, or multi-dimensional view of the data from a medical scanner. As a technical regulation, the reason of computer visualization is to achieve the building of computer visualization systems by using the theories and models.

Conventional machine visualization system applications take in the following aspects:

(1) Control process (for example, industrial robots or autonomous vehicles);

(2) Detection of an event (such as video surveillance or people counting);

(3) Organization information (such as index database of images and image sequences);

(4) Modeling objects or the environment (e.g., industrial inspection, medical image analysis or topographical modeling);

(5) Interaction (such as human-computer interaction input devices).

Very large applications of machine visualization in the image, such as the creation of 3-D computer graphics model data, frequently produce from the image data model through $3 \mathrm{D}$ aspects. Mainframe visualization is based on scene reconstruction, event detection, video tracking, target recognition, learning, indexing, motion estimation and image restoration Computer visualization is a diverse and moderately novel region. The most intimately connected to the field are image processing, image analysis and computer visualization.

These linked fields are built-in in the range of technologies and applications using the similar essential technology. Computer visualization tends to focus on one or more images. 


\section{COMPUter Visualization Method}

One of the most well-known application areas of computer visualization is medical image processing. The uniqueness of the request areas are the information extracted from a patient's medical diagnostic imaging data. usually, data may be from a microscopic image in the form of X-ray image or any another famous methods. The information can be extracted from these images, for example, detection of cancer arteriosclerosis, or other harmful changes. Data can also be from organ size, blood flow, and additional measuring, etc.

The major methods of computer visualization in medical image processing are classified as the following categories:

(1) Linear filtering;

(2) Principal component analysis;

(3) Independent component analysis;

(4) Hidden Markov model;

(5) Partial differential equations;

(6) Self- organization chart;

(7) Neural network;

(8) Wavelet.

With the development of computer application technology, new methods are emerging. The level set method is widely used in the field of machine visualization. It is mostly the add to of image, denoising, segmentation, repair, tracking outstanding advantages. Curve development method is an application which needs the incline of the image information for active contour model. The model can extract complex shape of the target, and the main outline of the initial contour. The sensitivity is also reduced. For overcome the shortcomings of the level set function depends signed distance function, a new variational formula is used to remove the re-initialization step so as to get better the velocity of the curve evolution. Finally, it minimise the time complexity of the level set function.

Medical liver images, using the methods of machine visualization, are processed in this paper through a novel approach. The approach is mainly separated into the following steps:

A Machine Visualization-based Medical Image Processing Cardiac medical images obtained by special machines, contain rich texture and complexity of the information presented in the form of the liver itself. The machine visualization-based medical image processing includes four steps which are detailed illustrated as follows.

\section{A.Step1: Smoothing}

The usual image smoothing algorithm compromises Gaussian smoothing, the mean smoothing and Gabor smoothing. Gaussian smoothing uses a Gaussian function. The method uses a Gaussian purpose to constitute a low-pass filter in the frequency domain.

\section{B.Step2: Mean Filtering}

The mean filtering algorithm is based on the proposed approaches from Duda and Hart. These approaches use error square dynamic clustering method to process the picture.

\section{Step 3: Enhancing}

Cardiac medical image texture has a riches data. Image enhancement not only straight relates to liver detail enhancement, but also considers the entire texture data outcome in a positive sense.

\section{Step 4: Restoration}

The image restore is the final stage. Image restoration algorithm is based on machine visualization, not only of the structural characteristics, but also for the texture information which has to been grasped. The tests are based on cardiac medical images. The proposed method has been adopted on processing these images. Matlab 2009 has been used for this purpose. 


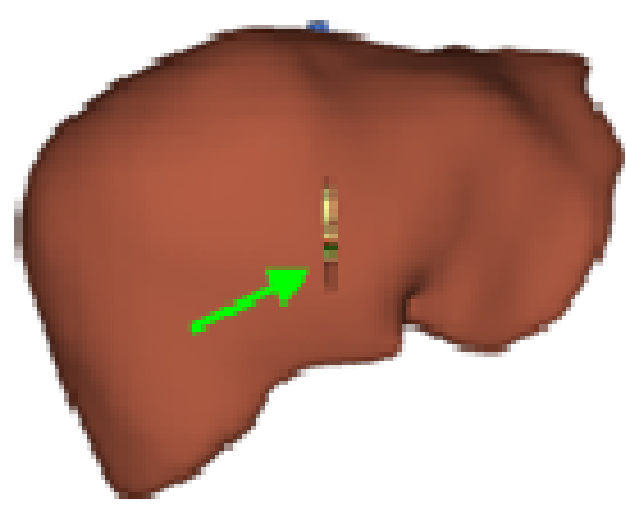

Figure1. The Original Image

\section{ReSUlts}

Using the planned smoothing and filtering algorithms, Figure (2) presents four images from different dimensions obtained after the smoothing and filtering of the projected algorithm for image processing. The above two is the images obtained following the smoothing algorithm. It can be seen that the image is positive and negative results after the smoothing which has minimized a lot of noises. In addition, the positive and negative for smoothing is because that all the information of the image of the liver may be presented, specially vascular and cardiac subtle texture. If the single always smooth used, some important information might be ignored.
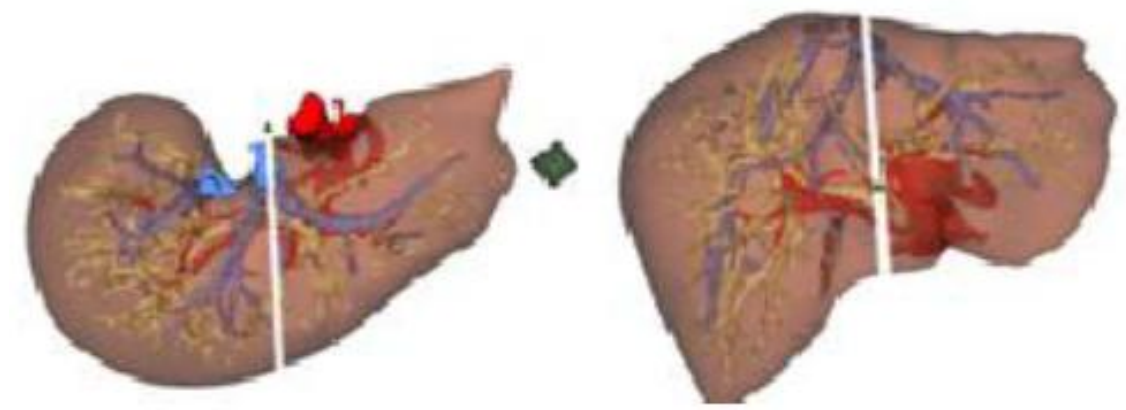

Figure2. Images after Smoothing and Filtering

The two images from the bottom in Figure 2 are color images after filtering. Compared with the on top of two figures, wherein the texture feature, specially arteries and major texture region are prominent features out. This is since the filtering not simply uses filtering algorithm pixel filter to proceed the image. And the squared mistakes and noise of the pixel cluster is measured. Thus, the center area of image texture characteristics is highlighted.

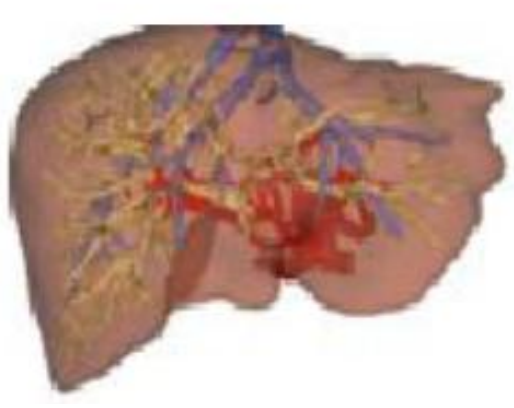

Figure 3 (a) Images after Enhancement;

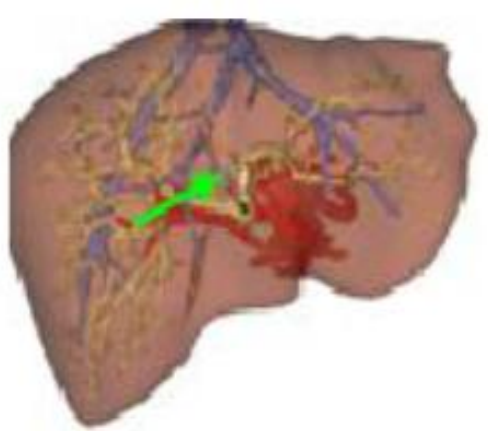

(b) Images after Restoration

As can be seen from the Figure (2), the disturbed regions are more concentrated following filtering through a description of the distance image. This methodology not only amplifies the information area, but also obtains more feature values from the observation. Figure 3 (a) shows a color effect after the image enhancement using the projected enhancement algorithm. It can be seen that the image focus areas and prominent feature 


\section{Conclusion}

This manuscript presents a methods for medical color image processing. This method has simple four steps. For the initial processing, the smoothing algorithm is to reduce noise. Then smooth the image is addressed by the filter algorithm which performs in the filtering process. The application of the image distance difference method is to boosted the filtering effect. then, the image enhancement algorithm applies weighted enhanced images of various regions. lastly, restoration algorithm is used to reinstate the color images of the liver. The experimental results show that the projected four-step method can be efficiently used to attain a good image expected effect.

\section{REFERENCES}

[1] A. Rosenfel, Computer vision, a source of models for biological visual process, IEEE Transaction on Biomedical 36(1), pp. 83-94, 1989.

[2] I. Sobel, Neighbourhood coding of binary images fast contour following and general array binary processing, Computer graphics and image processing vol. 8, pp. 127- 135, 1978.

[3] D.Marr, E.C.Hildreth. Theory of edge detection, proceeding of the Royal Society, 201b, pp187217, 1980. J.Canny. A computational approach to edge detection, IEEE Transactions in pattern analysis and machine intelligence vol. 8 pp. 679-698, 1986.

[4] M.H.Hueckel, An operator which locate edges in digitized pictures, Journal of ACM vol. 18, pp. 113-125, 1971.

[5] R.Maini, H.Aggarwal. Study and comparison of various image edge detection techniques, International Journal of Image processing (IJIP), volume (3), issue (1) 2009.

[6] N. Senthilkumaran and R.Rajesh, Edge Detection Techniques for image segmentation-A survey, Proceedings of the international conference on managing next generation software applications (MNGSA-08) pp. 749-760, 2008.

[7] N. Senthilkumaran and R.Rajesh, Edge detection Techniques for image segmentation-A survey of soft computing approaches, International Journal of Recent trends in Engineering, vol. 1 no 2, 2009.

[8] N. Senthilkumaran and R.Rajesh, A study on split and merge for region based image segmentation, proceedings of UGC sponsored national conference network security (NCNS-08) pp57-61, 2008.

[9] X.BIN Wen, H.Zheng and Z. Tao. Multiscale Unsupervised Segmentation of SAR Imagery using the generic algorithm, Sensors, vol8, pp. 1704-1711, 2008.

[10] M. Paulinas and A.Usnskas, A survey of generic algorithm application for image enhancement and segmentation, information technology and control vol. 36, no 3, pp. 278-284, 2007.

[11] R.C.Gonzalez and R.E.Woods, Digital Image Processing 2nd ed. Prentice Hall, 2002. R.A.Kirsch, Computer determination of the constituent structure of biomedical images, comput Eiorned.Res. Vol. 4, pp315-325 1971.

[12] Y. Yakimovsky. Boundary and object detection in real world images. Journal of ACM, vol. 23, no 4 pp. 598-619, 1976. 